\title{
Optical Observation of LEO Debris Caused by Feng Yun 1C
}

\author{
By Hirohisa KUROSAKI, Toshifumi YANAGISAWA and Atsushi NAKAJIMA \\ Aerospace Research and Development Directorate, Japan Aerospace Exploration Agency, Tokyo, Japan
}

(Received April 30th, 2008)

\begin{abstract}
Many pieces of space debris are in low earth orbit (LEO), and may be a serious problem in the near future. They are very hazardous to spacecraft such as the ISS, in which humans stay for long periods. In January 2007, China performed an experimental destruction of the meteorological satellite FengYun-1C in low earth orbit using a ballistic missile. Optical instruments for space debris observation were installed on Mt. Nyukasa in Nagano Prefecture by JAXA, and the resulting low earth orbit debris was observed with the small telescope there.

We have developed an image-processing technique, the line-detection method, to extract such effects as the streaks created by meteors, LEO satellites, and LEO debris. We succeeded in detecting the trajectories of specified FengYun-1C debris whose TLE were known. In this paper, the detection and observation of low earth orbit debris are discussed.
\end{abstract}

Key Words: Space Debris, LEO, Optical Observation, Telescope, CCD

\section{Introduction}

Artificial satellites that have finished their missions and used rockets are termed space debris, and most of them are not under any control. Space debris in low earth orbit goes around the earth at high speed. Such space debris undergoes mutual collisions, resulting in the proliferation of many smaller fragments of debris and explosions of residual fuel. This dramatically increases the quantity of space debris. The space debris problem must be solved for the sake of future space development and safety.

There are many pieces of space debris in low earth orbit (LEO) that may be a serious problem in the near future. These are particularly hazardous to spacecraft such as the ISS (International Space Station), in which humans remain for extended periods. In January 2007, China performed an experimental destruction of the meteorological satellite FengYun-1C in low earth orbit using a ballistic missile ${ }^{1)}$. This satellite was in a sun-synchronous orbit at an altitude of $850 \mathrm{~km}$. This destruction experiment generated tens of thousands of pieces of debris. The number of objects whose orbits were determined by observations exceeded 2100 by April 2008. When the low earth orbit debris is observed with an optical telescope system, it passes completely through the field of view of a CCD image in just several seconds, so a special method is necessary to detect it. This study examines a method of detecting debris trajectories from observed images. This method can detect faint trajectories that are too dark to be seen in the image.

\section{FengYun-1C Debris}

FengYun-1C, $2.02 \times 2.00 \times 2.215 \mathrm{~m}$ in size and weighing $958 \mathrm{~kg}$, was launched by a CZ-4B booster from Taiyuan on May 10, 1999 to a sun-synchronous $870 \mathrm{~km}$, 98.8 degree inclination orbit, the first of three such satellites to be fully operational. It remained fully functional until 2005.

\subsection{Anti-Satellite Experiment}

On January 11, 2007, China tested an anti-satellite (ASAT) weapon against this defunct Chinese weather satellite. This scattered thousands of fragments of various sizes into low earth orbit. This satellite's destruction is now viewed as the most serious fragmentation in the course of space operations. NASA estimates that the number of debris pieces larger than one centimeter is greater than $35,000^{2)}$. The ISS is in harm's way due to debris from the Chinese ASAT test. As this breakup took place at a high altitude where the atmospheric density is very low, a large fraction of the debris will remain in orbit for decades.

\section{Optical Obse rvation System}

Observations of satellites and debris in low earth orbit usually use the satellite tracking system at the Chofu aerospace center, JAXA (Chofu, Tokyo). However, in this case it seems that fragments are small because they are the result of deliberate destruction. Therefore, we observed them from the more suitable Nyukasayama optical observatory. The Nyukasayama optical observatory does not install the low earth orbit tracking system to put a weight point for the observation of the geosynchronous orbit debris mainly. Two telescopes of $35 \mathrm{~cm}$ and $25 \mathrm{~cm}$ are installed here. Two telescopes of $35 \mathrm{~cm}$ and $25 \mathrm{~cm}$ are each equipped with high-sensitivity CCD cameras ${ }^{3)}$.

\subsection{Nyukasayama Optical Observatory}

To develop GEO debris observation technology, an optical telescope has been installed at Nyukasayama Optical Observatory, Nagano prefecture. Figure 1 is a photo of the Nyukasayama Optical Observatory. This observation site has been in operation since last winter. The altitude of the site is $1,870 \mathrm{~m}$, in the northern area of 


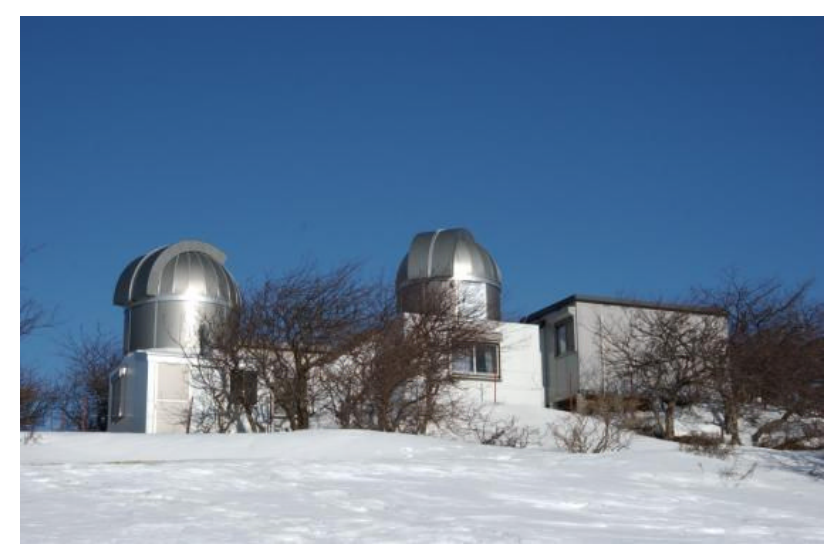

Fig. 1. Nyukasayama Optical Observatory Lat. $35^{\circ} 54^{\prime} 05^{\prime} \mathrm{N}$, Long. $138^{\circ} 10^{\prime} 18^{\prime}$ 'E, Elev.1,870 m

the Japanese South Alps.

\subsection{Observation System}

This observatory will consist of two 3-meter domes for $35 \mathrm{~cm}$ and $25 \mathrm{~cm}$ telescopes with equatorial mounts, a $2 \mathrm{~K} 2 \mathrm{~K}$ back-illuminated CCD camera cooled by peltier devices and a 4K4K CCD camera cooled by refrigeration. The image sensors used at this facility are back-illuminated high-speed readout CCD cameras. Figure 2 is a photograph of the $35 \mathrm{~cm}$ telescope and the $2 \mathrm{~K} 2 \mathrm{~K}$ CCD camera. Tables 1 and 2 give overviews of the main characteristics of the $35 \mathrm{~cm}$ telescope and the $2 \mathrm{~K} 2 \mathrm{~K}$ CCD camera, which is currently the main detector at this facility. Figure 3 is $25 \mathrm{~cm}$ Telescope and others. We tried several observation techniques, as explained below.
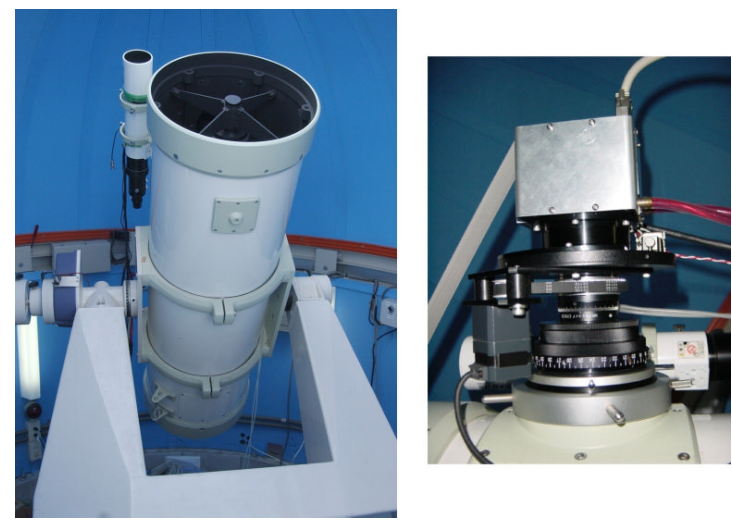

Fig. 2. $35 \mathrm{~cm}$ Telescope and $2 \mathrm{~K} 2 \mathrm{KCCD}$ camera

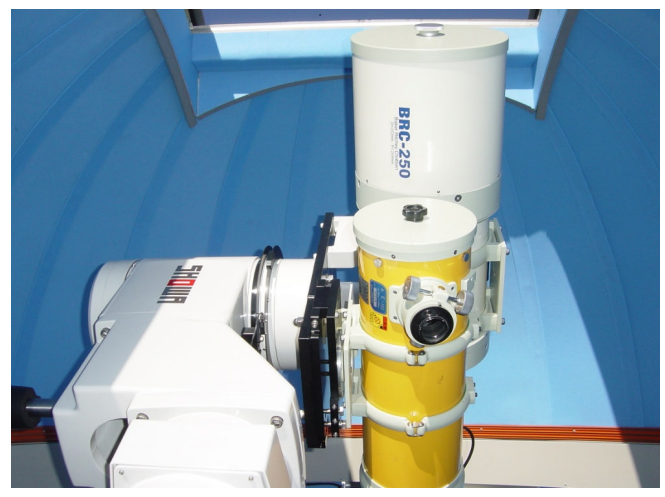

Fig. 3. $25 \mathrm{~cm}$ and other Telescopes
Table 1 Characteristics of the $35 \mathrm{~cm}$ Telescope and Mount System

\begin{tabular}{|l|l|}
\hline Telescope & $\boldsymbol{\varepsilon}-350$ (Takahashi Co.) \\
\hline Diameter & $350 \mathrm{~mm}$ \\
\hline Focal Length & $1,248 \mathrm{~mm}(\mathrm{~F} / 3.6)$ \\
\hline Image circle & $70 \mathrm{~mm}$ \\
\hline Mount & $\begin{array}{l}\text { SHOWA 25EF Equatorial Fork- } \\
\text { Mount (Showa Co.) }\end{array}$ \\
\hline Drive & Stepping Motor $(85 \mathrm{deg} / 60 \mathrm{sec})$ \\
\hline
\end{tabular}

Table 2 Characteristics of the $2 \mathrm{~K} 2 \mathrm{~K}$ CCD Camera

\begin{tabular}{|l|l|}
\hline CCD camera & e2v CCD42-40 Back-illuminated \\
\hline Pixels & $2048 \times 2048$ \\
\hline Pixel size & $13.5 \mu \mathrm{m} \times 13.5 \mu \mathrm{m}$ \\
\hline Area & $27.6 \mathrm{~mm} \times 27.6 \mathrm{~mm}$ \\
\hline FOV (with 35cm Tele.) & $1.27 \times 1.27 \mathrm{deg}$. \\
\hline Readout time & $10 \mathrm{sec}(2 \mathrm{ch}$ out $)$ \\
\hline Exposure time & $1-7200 \mathrm{sec}$ \\
\hline A/D trans. & $1 \mathrm{MHz}-50 \mathrm{kHz}($ Variable $)$ \\
\hline Cooling & Peltier + water cooling \\
\hline Shutter & Copal DC-392 (Mechanical) \\
\hline Power supply & DC12, $2 \mathrm{~A}$ \\
\hline Dimensions & W140 $\times$ H140 $\times$ D100 mm \\
\hline Mass & $1.5 \mathrm{~kg}$ \\
\hline
\end{tabular}

\section{Observation techniques}

\subsection{Standby observation from TLE}

This method observes a field of view in the sky calculated from the TLE of targeted FengYun-1C debris so that a track of the target is obtained.

The average orbital element that was expressed with such an earth center inertia coordinate system that calculate the orbit of the space object is shown with a format called TLE. This is the format used by NASA to distribute satellite elements in their NASA Prediction Bulletin. A two line element set (TLE) is a group of numbers provided in two lines and separated by spaces that specify the orbital parameters (elements) for a satellite, spent booster rocket, or other artificial satellites. The exposure is begun immediately before the targeted debris passes through the field of view.

We tried this method for a few days at the end of January, 2007, and detected about thirty pieces of debris. It seems that comparatively large and bright debris was detected by this method. The observation used the $35 \mathrm{~cm}$ telescope and the $2 \mathrm{k} 2 \mathrm{kCCD}$ camera. Figure 4 shows FengYun-1C DEB (99025AJ), observed on January 24, 2007. Figure 5 shows FengYun-1C DEB (99025AD), observed on January 25, 2007. Streaks of light are recorded in these images. The positions and tilts of both the rays and the observed times are in good agreement with forecasts from the orbital elements, convincing us that the observed streaks are the targets.

\subsection{Observation by the field of view fix}

This method repeatedly takes images of the same field of view in the sky. When FengYun-1C debris passes 
through the field of view by chance, it is recorded on the image. A huge amount of data is needed in order to detect such a rare event. The method is used to detect unknown FengYun-1C debris that may have no known TLE elements and that will mostly be faint. We observed with this method for a few nights in November, 2007. Table 3 shows observational dates and times. We obtained images of some fields of view consecutively with 10 seconds exposures. The readout time of the CCD camera is about 10 seconds. Visual inspection of these images revealed no FengYun-1C debris. However, even when no visible event is detected, there may be faint events that are almost invisible in the images, and we have image processing techniques to detect such faint events. The detail of the techniques and the results from their application are described in section 5 .

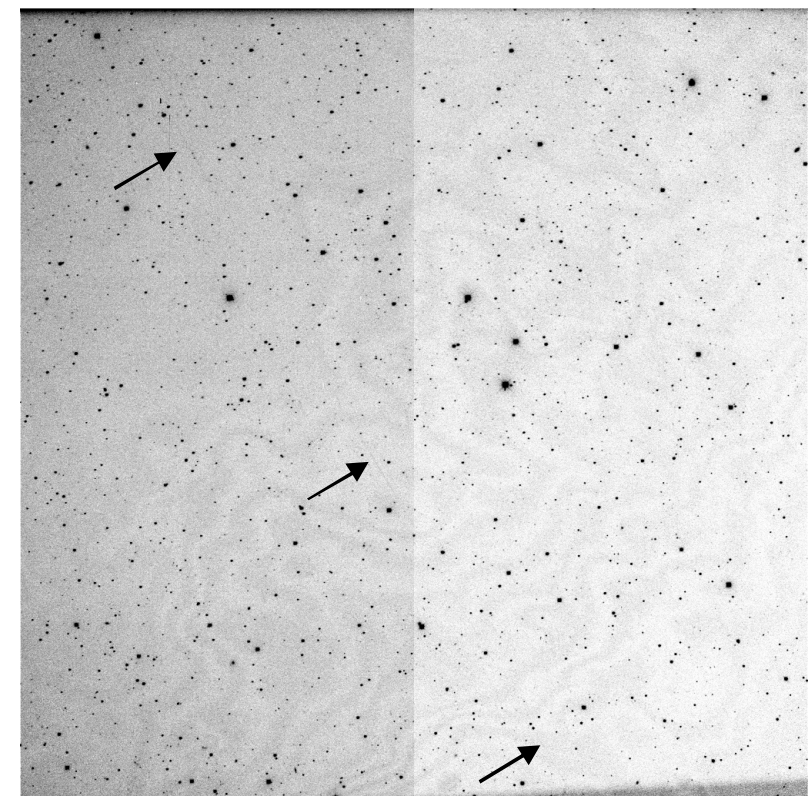

Fig. 4. Image of Feng Yun-1C DEB (99025AJ)

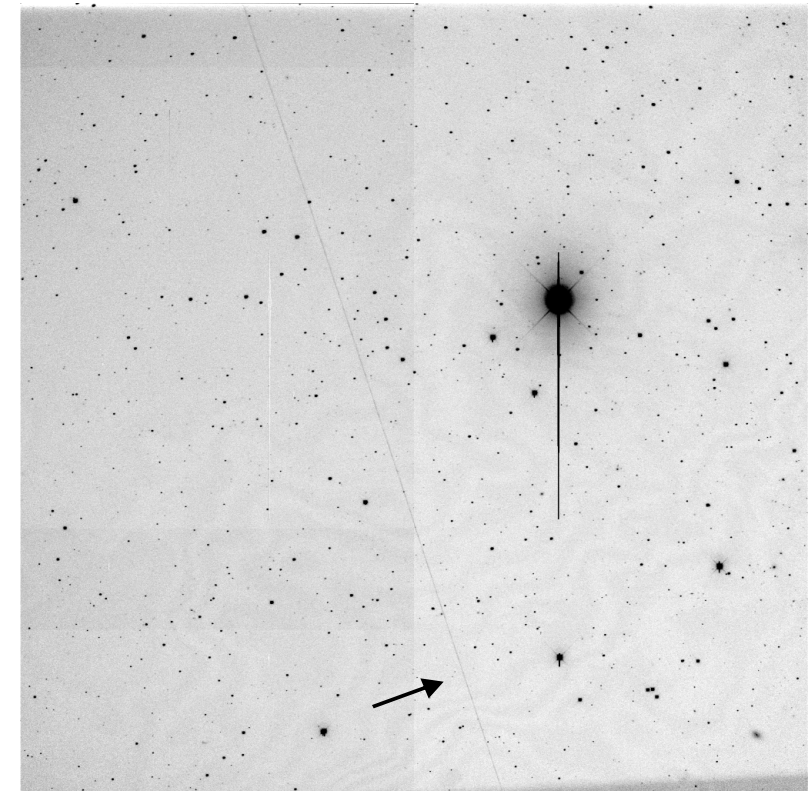

Fig. 5. Image of Feng Yun-1C DEB (99025AD)

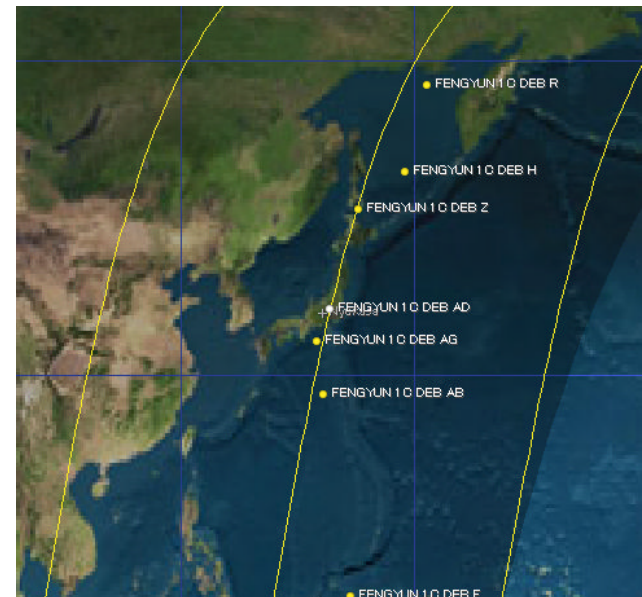

Fig. 6. Orbit of FengYun-1C (99025AD)

Table 3 Observation area of Feng Yun-1C debris

\begin{tabular}{|c|c|c|c|}
\hline DATE & $\begin{array}{l}\text { Observation point } \\
\text { R.A. Decl. }\end{array}$ & $\begin{array}{l}\text { Telescope } \\
\text { Camera }\end{array}$ & Frame \\
\hline 2007.11 .7 & $\begin{array}{l}11 \mathrm{~h} 19 \mathrm{~m} 43 \mathrm{~s}+17 \mathrm{~d} 30 \mathrm{~m} 37 \mathrm{~s} \\
09 \mathrm{~h} 22 \mathrm{~m} 08 \mathrm{~s}+32 \mathrm{~d} 13 \mathrm{~m} 48 \mathrm{~s} \\
08 \mathrm{~h} 24 \mathrm{~m} 37 \mathrm{~s}+51 \mathrm{~d} 27 \mathrm{~m} 59 \mathrm{~s}\end{array}$ & $25 \mathrm{~cm} 2 \mathrm{~K} 2 \mathrm{~K}$ & $\begin{array}{l}100 \\
100 \\
100\end{array}$ \\
\hline 2007.11 .8 & $07 \mathrm{~h} 18 \mathrm{~m} 05 \mathrm{~s}+55 \mathrm{~d} 28 \mathrm{~m} 46 \mathrm{~s}$ & $25 \mathrm{~cm} 2 \mathrm{~K} 2 \mathrm{~K}$ & 240 \\
\hline 2007.11 .14 & $07 \mathrm{~h} 32 \mathrm{~m} 05 \mathrm{~s}+55 \mathrm{~d} 28 \mathrm{~m} 48 \mathrm{~s}$ & $35 \mathrm{~cm} 2 \mathrm{~K} 2 \mathrm{~K}$ & 150 \\
\hline 2007.11 .15 & $07 \mathrm{~h} 32 \mathrm{~m} 05 \mathrm{~s}+55 \mathrm{~d} 28 \mathrm{~m} 46 \mathrm{~s}$ & $25 \mathrm{~cm} 2 \mathrm{~K} 2 \mathrm{~K}$ & 270 \\
\hline
\end{tabular}

\section{The line-detection method}

Meteors, LEO satellites and LEO debris leave streaks on a CCD image when they pass through the field of view of the CCD camera. If they are too faint, the streaks are not recognized on the CCD images. However, their signals must be there, even though they are buried in the sky background noise. We developed an image-processing technique, the line-detection method, to extract such streak images of meteors, LEO satellites, and LEO debris ${ }^{4)}$.

\subsection{Details of the method}

Figure 7 shows the procedure for the line-detection method. Figure 7 (a) is a raw CCD image. The white spots are fixed stars. In this image, no lines are observable at first inspection. Fixed stars are first removed by subtracting a sequent image (figure 7 (b)). The image is then rotated by a suitable angle, and the effective region unaffected by the rotation process is cut out (figure 7 (c)). Finally, median values of all pixels of every line along the arrow are calculated. The last two parts of the process (figure 7 (c) and (d)) are repeated for every angle of interest to detect weak, invisible lines. From the rotation angle and the coordinates of the effect, the actual position of the detected line is determined, as shown in figure 7 (e).

Figure 8 shows an example of a line effect detected in an image by using the line-detection method. The horizontal and vertical axes show the pixel numbers perpendicular to one direction and the values of each pixel 


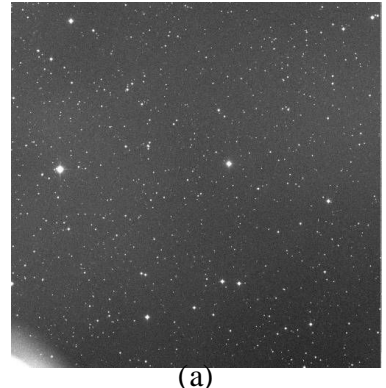

(a)

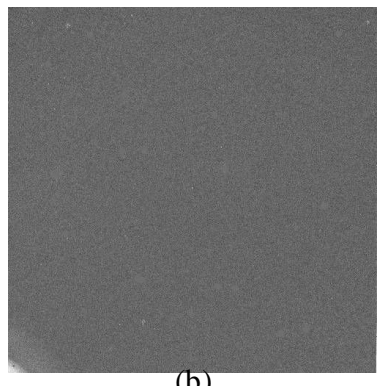

(b)

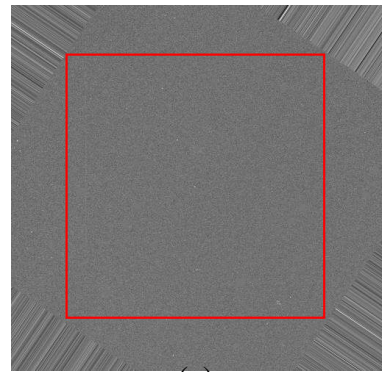

(c)

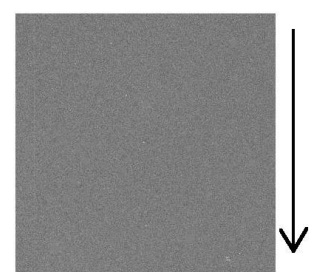

(d)

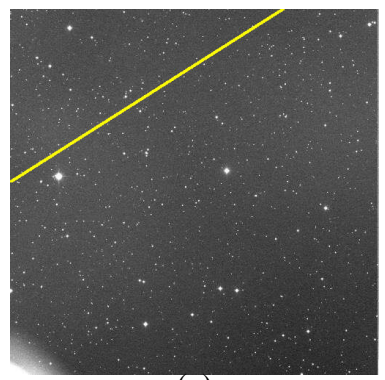

(e)

Fig. 7. Procedure for the line detection method.

in analog-to-digital units (ADU), respectively. Constant values were added to the upper two lines for clear presentation. The bottom line shows the noise level of a raw CCD image. The middle line shows the median values of every 20 pixels along the direction of the line. The line effect is visible at the center. The top line shows the median values of all pixels along the line direction. The effect is clearly confirmed. This effect appears only when the line direction corresponds exactly with the rotation angle.

\subsection{Results}

Images acquired by the observations were analyzed by this method. When an orbit was calculated from some debris, it was found that FengYun-1C debris should pass through the image at an angle of 6 degrees. The image was rotated by 6 degrees to simplify image processing, and all pixels in a vertical line were summed. The rotation angle of the image was incremented in 0.1 degree steps. The result records the one with more than $3 \sigma$ as an event. $\sigma$ is standard deviation of the sky level. Of course, it is guessed that there is a splinter of FengYun1C though there is a possibility of other objects, too. The result records any sum that was more than $3 \sigma$ from background as an event, probably (though not necessarily) due to a splinter of FengYun1C. Figure 9 shows the analyzed

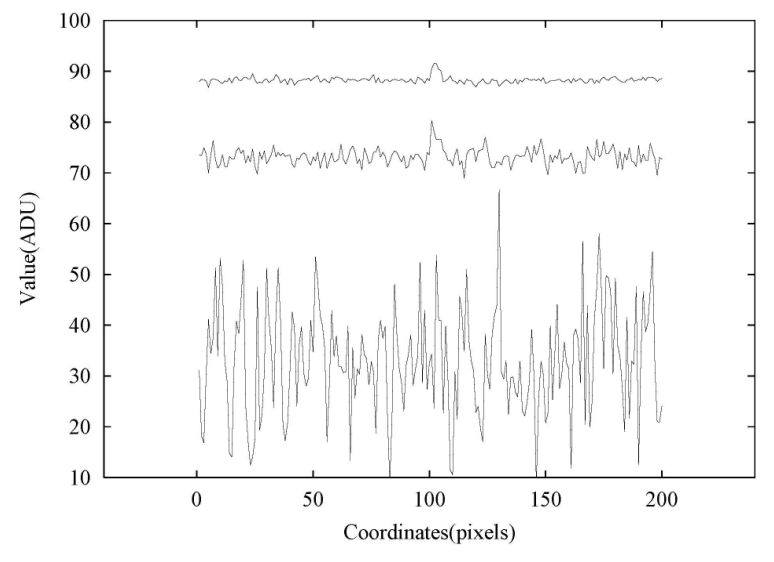

Fig. 8. Sample result of the line-detection method.

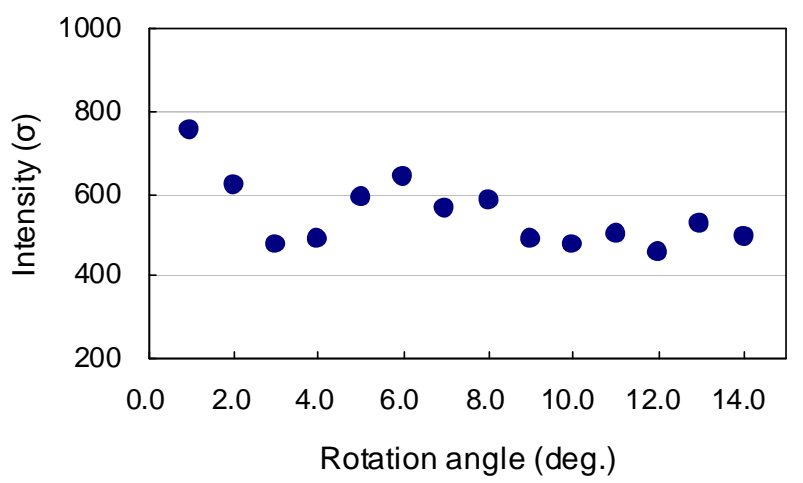

Fig. 9. Graph of rotation angle and intensity of signal.

results of the data of November 15 . The $x$ - and $y$-axes represent the rotation angle and the sum total of the $\sigma$ value, respectively. The expected tilt angle for FengYun-1C debris is from six to seven degrees, and a slight increase in intensity is seen around that angle. There is influence of the dispersion of a vertical transfer line of the CCD in less than 2 degrees angle of rotation.

\subsection{Detection Size}

Next, the size (brightness) of the debris detectable by this method was examined. Magnitude and the relations of the flux (ADU) are given from image (1). Altitude of orbit is assumed to be $855 \mathrm{~km}$, and time to pass image is calculated (2). Debris was assumed to be $5 \sigma$, and the median was taken, and it was calculated with 11.37 mag. when magnitude of the debris was demanded using $\sigma$, (1), (2). When debris $10 \mathrm{~cm}$ diameter debris of the geosynchronous orbit (about 36,000 km) is assumed to be the 20th magnitude class, a size of the debris of 11.37 mag. is $13 \mathrm{~cm}$ at an altitude of $855 \mathrm{~km}$.

\section{Conclusion}

There are many pieces of space debris in low earth orbit (LEO), and they may be a serious problem in the near future. In January 2007, China performed an experimental destruction of the meteorological satellite (FengYun-1C) in low earth orbit using a ballistic missile.

We carried out observations on a number of nights to 
capture trajectories of the FengYun-1C debris and analyzed the images obtained. We succeeded in detecting the trajectories of specified FengYun-1C debris whose TLE were known. For the unknown debris, the increase around the expected angle for FengYun-1C debris was recognized. Significant amounts of otherwise unrecognized debris were also found on the expected trajectory of FengYun-1C debris, using our image processing technique.

\section{References}

1) NASA Orbital Debris Quarterly News, NASA, Vol.11,2 (2007).

2) NASA Orbital Debris Program Office

3) H. Kurosaki, et al.: Development of Detection Technology for Space Debris in Nyukasayama Optical Observatory, JSASS, 51st Space Sciences and Technology Conference, 2F16 (2007).

4) T. Yanagisawa, et al.: High-Precision Radiant Analysis of the 2001 Leonids using Telescopic Optics, Publications of the Astronomical Society of Japan, 55 (2003), pp.553-557. 